\title{
Pharmacological strategies to prevent haemodynamic changes after intubation in parturient women with hypertensive disorders of pregnancy: A network meta-analysis
}

\author{
Sang Won Yoon ${ }^{1 \#, ~ G e u n ~ J o o ~ C h o i ~}{ }^{1 \#}$, Hee-Kyeong Seong${ }^{1}$, Myeong Jong Lee², Hyun Kang ${ }^{1 凶}$ \\ 1. Department of Anaesthesiology and Pain Medicine, Chung-Ang University College of Medicine, Seoul, Republic of Korea \\ 2. Department of Anaesthesiology and Pain Medicine, Konkuk University Medical School, Chungju, Republic of Korea \\ "Sang Won Yoon and Geun Joo Choi equally contributed to this study \\ $\triangle$ Corresponding author: Hyun Kang, M.D., Ph.D., M.P.H., Professor, Department of Anaesthesiology and Pain Medicine, Chung-Ang University College of \\ Medicine, 84 Heukseok-ro, Dongjak-gu, Seoul, 06911, Republic of Korea. Tel: +82-2-6299-2571, 2579, 2586; Fax: +82-2-6299-2585; E-mail: roman00@naver.com \\ (C) The author(s). This is an open access article distributed under the terms of the Creative Commons Attribution License (https://creativecommons.org/licenses/by/4.0/). \\ See http:/ /ivyspring.com/terms for full terms and conditions.
}

Received: 2020.10.02; Accepted: 2020.12.22; Published: 2021.01.01

\begin{abstract}
Objective: This network meta-analysis (NMA) aimed to determine the relative efficacy and safety of pharmacological strategies used to mitigate haemodynamic instability by intubation for general anaesthesia in hypertensive parturient women undergoing caesarean section.

Methods: We considered randomised controlled studies comparing the effects of pharmacological strategies used to alleviate haemodynamic instability during intubation in parturient women with hypertensive disorders of pregnancy. The primary endpoints were maximum blood pressure and heart rate after intubation, and secondary endpoints were the Apgar scores at 1 and $5 \mathrm{~min}$. NMA allowed us to combine direct and indirect comparisons between strategies.

Results: Twelve studies evaluating nine pharmacological strategies in 619 patients were included. According to the surface under the cumulative ranking curve, the maximal mean arterial pressure was lowest for high-dose remifentanil $(99.4 \%)$ followed by nitroglycerin $(73.6 \%)$ and labetalol $(60.9 \%)$. The maximal heart rate was lowest for labetalol (99.9\%) followed by high dose of remifentanil $(81.2 \%)$ and fentanyl (61.6\%). Apgar score at $1 \mathrm{~min}$ was higher with low-dose than with high-dose remifentanil (mean difference, 0.726 ; $95 \%$ confidence interval, 0.056 to $1.396 ; 12=0.0 \%$ ).

Conclusions: High-dose remifentanil produces minimum blood pressure changes, while labetalol is most effective in maintaining normal heart rate in parturient women with hypertensive disorders of pregnancy during caesarean section under general anaesthesia.
\end{abstract}

Key words: Caesarean section; pregnancy-induced hypertension; intubation; pregnancy

\section{Introduction}

Intubation during the induction period in general anaesthesia activates the sympathetic nervous system, resulting in haemodynamic instability, including hypertension and tachycardia [1]. These reflex haemodynamic responses are exaggerated in patients with hypertension history and constitute major concerns for anaesthesiologists [2-4]. For patients with hypertensive disorders of pregnancy, such as preeclampsia and gestational hypertension, intubation is often circumvented during caesarean section by performing local anaesthesia. However, due to certain conditions present in preeclampsia, including coagulopathy and thrombocytopenia, general anaesthesia is unavoidable in some patients. These patients exhibit a marked increase in blood pressure and heart rate during intubation and airway manipulation, which may result in elevated intracranial pressure, cerebral haemorrhage, cardiac 
failure, and pulmonary oedema [5]. These events increase the risk of morbidity and mortality for both the mother and child.

Several types of medication have been used by anaesthesiologists to mitigate the haemodynamic reflex after airway manipulation in parturient women with hypertensive disorders of pregnancy. Opioids such as remifentanil, fentanyl, and alfentanil, and antihypertensive drugs such as nitroglycerin, labetalol, and hydralazine have been prescribed, and their effectiveness and efficacy in controlling blood pressure and heart rate with minimal effects on foetuses have been compared. Numerous studies have investigated the most effective drugs that ensure haemodynamic stability without compromising the foetus in patients with preeclampsia. However, each study was limited to the comparison of two or three drugs, and the results were inconsistent.

Therefore, we reviewed all articles that compared the effects of different drugs used to alleviate haemodynamic instability during intubation in parturient women with hypertensive disorder of pregnancy, and conducted a network meta-analysis (NMA). NMA supplements traditional meta-analysis by combining both direct and indirect comparisons between pharmacological interventions, and generates an intervention ranking for each endpoint. Our primary endpoints were the maximum changes in blood pressure and heart rate after intubation, and the secondary endpoints were the Apgar scores of newborns at 1 and $5 \mathrm{~min}$.

\section{Methods}

We developed the protocol for this systematic review and NMA according to the preferred reporting requirements for systematic review and meta-analysis protocol (PRISMA-P) statement [6]. We registered the protocol in the PROSPERO network (registration number: CRD42019136067; www.crd.york.ac.uk/ prospero) on 02 June 2019, and published it in a peer-reviewed journal [7]. This study was performed according to the protocol recommended by the Cochrane Collaboration [8], and reported according to the PRISMA extension for NMA guidelines [9].

\section{Eligibility Criteria}

We included RCTs comparing two or more pharmacological strategies. The PICO-SD information was as follows:

(1) Patients (P): Parturient women with hypertensive disorders of pregnancy undergoing caesarean section under general anaesthesia; those undergoing surgery under regional anaesthesia were excluded.

(2) Intervention (I): Pharmacological strategies to prevent haemodynamic changes after intubation for general anaesthesia

(3) Comparison (C): other pharmacological strategies, placebo, or no treatment

(4) Outcome measurements $(\mathrm{O})$ :

(1) Effectiveness

Primary endpoints were maximal mean arterial pressure (MMAP) and maximal heart rate (MHR) after intubation. Maximal systolic arterial pressure (MSAP) and diastolic arterial pressure (MDAP) were also assessed. MMAP, MHR, MSAP and MDAP after intubation were considered as the maximum value of the endpoints, not maximum change of them.

(2) Safety

Apgar scores at 1 and 5 min after delivery were assessed.

(5) Study design (SD): We included peer-reviewed RCTs without language or date limitations. Review articles, case reports, case series, letters to the editor, commentaries, proceedings, laboratory science studies, and other non-relevant studies were excluded.

\section{Information Sources}

We searched MEDLINE, Embase, the Cochrane Central Register of Controlled Trials (CENTRAL), and Google Scholar, from their launch to November 2019, using search terms such as, and related to, 'pregnancy induced hypertension', 'caesarean section' and 'haemodynamic change'. The search strategy, which included a combination of free text, Medical Subject Heading (MeSH) terms, and EMTREE terms, is outlined in the Supplemental Digital Content. Two authors (SWY and GJC) screened the titles and abstracts of the retrieved articles. Reference lists were imported to Endnote software 8.1 (Thompson Reuters, CA, USA) and duplicate articles were removed. Additional relevant articles were identified by scanning the reference lists of articles obtained from the original search.

\section{Study Selection}

Titles and abstracts were reviewed independently by two investigators. To minimise data duplication due to multiple reporting, papers from the same author, organisation, or country were compared. For articles determined to be eligible based on the title or abstract, the full paper was retrieved. All abstracts not providing sufficient information regarding the eligibility criteria were selected for full-text evaluation. Potentially relevant studies chosen by at least one investigator were retrieved and the full text was evaluated. Articles meeting the inclusion criteria were assessed separately by two authors (SWY and GJC), and disagreements were 
resolved through discussion or with the help of a third investigator (HK).

The degree of agreement between the two authors was calculated using the kappa statistics. Kappa values were interpreted as follows: less than 0 : no agreement; 0.01-0.20: slight agreement; 0.21-0.40: fair agreement; 0.41-0.60: moderate agreement; 0.610.80: substantial agreement; and 0.81-1.00: almost perfect agreement [10].

\section{Data Extraction}

Using a standardised extraction form, the following data were extracted independently by two investigators (SWY and GJC) and then cross-checked: (1) title; (2) author names; (3) journal name; (4) publication year; (5) study design; (6) clinical trial registration; (7) competing interests; (8) country; (9) risk of bias; (10) number of patients; (11) drugs and doses compared; (12) age of parturient women; (13) weight of parturient women; (14) height of parturient women; (15) duration of anaesthesia; (16) American Society of Anaesthesiologists' physical status score; (17) inclusion criteria; (18) exclusion criteria; (19) drugs used for induction; (20) MSAP; (21) MMAP; (22) MDAP; (23) MHR; and (24) Apgar scores. Disagreements were resolved with the aid of a third investigator (HK).

Data were extracted from the tables or text. Missing information was calculated from the available data, or extracted from the figures using the open source software Plot Digitizer (version 2.6.8; http://plotdigitizer.sourceforge.net). Because the studies comparing the effect of remifentanil used different doses, remifentanil groups were categorised into low-dose $(<1 \mathrm{mcg} / \mathrm{kg})$ and high-dose $(\geq 1$ $\mathrm{mcg} / \mathrm{kg}$ ).

\section{Study Quality Assessment}

The quality of the studies was independently assessed by two authors (MJL and $\mathrm{HK}$ ) using the Revised Cochrane risk of bias tool for randomised trials (RoB 2.0) [11]. The risk of bias (ROB) was evaluated by considering the following domains: (1) bias arising from the randomisation process; (2) bias owing to departures from the intended interventions; (3) bias from missing outcome data; (4) bias in measurement of the outcome; and (5) bias in selection of the reported results, including deviations from the registered protocol. The response options for each risk of bias judgement were 'low risk of bias', 'some concerns', and 'high risk of bias'.

The overall ROB was evaluated according to these domain-level judgements [11]. We rated trials at: (1) low risk of bias overall if all domains were rated as low risk of bias; (2) some concerns overall if at least one domain was rated as some concerns; and (3) high risk of bias overall if at least one domain was rated as high risk of bias or some concerns for multiple domains in a way that substantially lowered confidence in the result.

\section{Statistical Analysis}

Ad-hoc tables were designed to summarise data from the studies and show their key characteristics and important questions related to the review objectives. After extracting the data, we determined the feasibility of a meta-analysis. Specifically, we evaluated the heterogeneity and transitivity assumptions by examining the comparability of eligibility criteria and patient demographics and the ROB as potential treatment-effect modifiers across comparisons [12].

When the treatment nodes formed a connected network of evidence, we performed NMA. A multiple treatment comparison NMA, which is a generalisation of methods used in meta-analysis, includes both direct and indirect comparisons between treatments. An NMA based on a frequentist framework was performed using the NMA graphical tools by Chaimani et al. [13]. Given the heterogeneity of populations and methods among the included trials, we used the random-effects model in our primary analysis.

A network plot linking all included pharmacological strategies was created to indicate the type of pharmacological strategy, number of patients under different pharmacological strategies, and number of pair-wise comparisons. The nodes of the network plot indicate the pharmacological strategies being compared, while the edges indicate the available direct comparisons between pharmacological strategies. Each drug or drug combination was treated as a node in this network. Nodes and edges were weighted on the basis of the number of parturient women and the inverse of the standard error of the effect.

Contribution plots were used to represent the percent contribution of each estimate in the summary estimate and the entire network. We displayed the contribution percentage of each comparison by weighted squares in the contribution plots.

We examined the consistency of the total network through global and local tests of inconsistency. We evaluated the global consistency assumption using the design-by-treatment interaction model [14]. We also evaluated each closed loop in the network to examine local inconsistencies between direct and indirect effect estimates for the same comparison. In each loop, we estimated the inconsistency factor (IF) as the absolute difference 
(with 95\% confidence interval [CI] and a z-test) between direct and indirect estimates for each paired comparison. The IF is the logarithm of the ratio of two odds ratios (RoR) from the direct and indirect evidence in the loop; RoR values close to 1 indicate agreement.

We also showed the relative treatment effects between all active pharmacological strategies using ranked forest plots. The mean summary effects with CIs are presented together with their predictive intervals to facilitate the interpretation of the results in light of the magnitude of heterogeneity. Predictive intervals provide an interval expected to encompass the estimate of a future study.

Rankograms and cumulative ranking curves were drawn for each pharmacological strategy. A rankogram plots the probabilities for treatments to assume any possible rank among all treatments evaluated in the NMA. We used the surface under the cumulative ranking curve (SUCRA) value to present the hierarchy of pharmacological strategies. The SUCRA is a relative ranking measurement that accounts for the uncertainty in the treatment order, by taking into account both the location and variance of all relative treatment effects [15]. The SUCRA can assume values up to $100 \%$, with higher values suggesting better pharmacological strategies.

We tested for small study effects and publication bias using the comparison-adjusted funnel plot [16]. As the number of included studies was fewer than 10 for all outcomes except MHR, publication bias was not assessed for these outcomes.

If only two groups were compared for certain outcomes, a pair-wise meta-analysis was conducted to generate summary estimates and assess statistical heterogeneity across the included studies. Summary estimates were reported as mean differences (MDs), standardised mean differences, or relative risks, as appropriate, with the corresponding 95\% CIs. Heterogeneity between studies was assessed using the Cochran's $Q$ and the Higgins $I^{2}$ statistics. A level of $10 \%$ significance $(\mathrm{P}<0.10)$ in the chi-squared statistics or an $\mathrm{I}^{2}$ greater than $50 \%$ indicated considerable heterogeneity, and the corresponding data were analysed using the Mantel-Haenszel random-effects model; otherwise, the MantelHaenszel fixed-effects model was applied [17]. All statistical analyses were performed using Stata SE, version 15.0 (StataCorp, College Station, TX).

\section{Evidence Synthesis}

The evidence grade was determined using the guidelines of the Grading of Recommendations, Assessment, Development, and Evaluation (GRADE) system, which uses a sequential assessment of evidence quality followed by an assessment of the risk-benefit balance and a subsequent judgement on the strength of the recommendation [18]. Two authors (MJL and HK) with experience in using GRADE rated each domain for each comparison separately and resolved discrepancies by consensus. We rated the certainty for each comparison and outcome as high, moderate, low, or very low, based on considerations of risk of bias, inconsistency, indirectness, imprecision, and publication bias.

\section{Results}

\section{Study Selection}

From the MEDLINE, Embase, CENTRAL, and Google Scholar database search, 93 studies were initially evaluated, and a subsequent manual search revealed five additional studies. After adjusting for duplicates, 95 studies remained. Of these, 79 were excluded after reviewing the titles and abstracts. The remaining 16 studies were reviewed in detail, after which four studies were excluded because of their retrospective nature [19] or because they did not report any outcome of interest [20-22]. Thus, 12 studies $[7,23-33]$ were eventually included in this systematic review and meta-analysis (Figure 1). The kappa value for selecting articles between the two reviewers was 0.826 .

\section{Study Characteristics}

The characteristics of the 12 RCTs that met the inclusion criteria are summarised in Table 1 . Studies were conducted in the USA [23, 24], South Africa [25-27], Republic of Korea [7, 28, 29], India [30, 31], and Iran $[32,33]$.

The pharmacological strategies applied to prevent haemodynamic changes after intubation in parturient women with hypertensive disorders of pregnancy included the administration of lidocaine [25], magnesium sulphate [25, 26], esmolol [30], labetalol [24], nitroglycerin [23,33], nifedipine [31,33], hydralazine [33], fentanyl [27, 32], alfentanil [25, 27], remifentanil [28, 29, 32, 34], combination of magnesium sulphate and alfentanil [26], and combination of esmolol and lidocaine [30] (Table 1).

\section{Study Quality Assessment}

The ROB assessment performed with the Cochrane tool for the included studies is presented in Table 2.

\section{Result Presentation}

For all outcomes, we presented the network plot (Figure 2), confidence interval plot (Figure 3), and expected mean ranking and SUCRA values for each pharmacological agent (Figure 4). Contribution plots, 
inconsistency plots between direct and indirect effect estimates for the same comparison, rankograms, and cumulative ranking curves are shown in Supplemental Figures S1, S2, S3, and S4, respectively. Apgar scores at 1 and $5 \mathrm{~min}$ in the network plot and confidence interval plot are shown in Supplemental Figures S5 and S6. Additionally, league tables of estimated effects of pharmacological strategies in network meta-analysis are shown in Supplemental Figure S7.

Table 1. Summary of randomised controlled trials included in the network meta-analysis

\begin{tabular}{|c|c|c|c|c|c|}
\hline $\begin{array}{l}\text { First author, } \\
\text { year }\end{array}$ & Country & Medication & Dosage & No. & Endpoints \\
\hline \multirow{3}{*}{$\begin{array}{l}\text { Allen RW, } \\
1991^{25}\end{array}$} & South & Lidocaine & $1.5 \mathrm{mg} / \mathrm{kg}$ & 21 & \multirow[t]{3}{*}{ SAP } \\
\hline & Africa & Alfentanil & $10 \mathrm{mcg} / \mathrm{kg}$ & 24 & \\
\hline & & Mg sulphate & $40 \mathrm{mg} / \mathrm{kg}$ & 24 & \\
\hline \multirow{2}{*}{$\begin{array}{l}\text { Ashton WB, } \\
1991^{26}\end{array}$} & South & Mg sulphate & $40 \mathrm{mg} / \mathrm{kg}$ & 19 & \multirow[t]{2}{*}{ SAP } \\
\hline & Africa & $\begin{array}{l}\text { Mg sulphate } \\
\text { +alfentanil }\end{array}$ & $30 \mathrm{mg} / \mathrm{kg}+7.4 \mathrm{mcg} / \mathrm{kg}$ & 19 & \\
\hline \multirow{4}{*}{$\begin{array}{l}\text { Bansal S, } \\
2005^{30}\end{array}$} & India & Esmolol & $1 \mathrm{mg} / \mathrm{kg}$ & 20 & \multirow{4}{*}{$\begin{array}{l}\text { SAP, HR, } \\
\text { Apgar }\end{array}$} \\
\hline & & Esmolol & $2 \mathrm{mg} / \mathrm{kg}$ & 20 & \\
\hline & & $\begin{array}{l}\text { Esmolol + } \\
\text { lidocaine (1) }\end{array}$ & $1 \mathrm{mg} / \mathrm{kg}+1.5 \mathrm{mg} / \mathrm{kg}$ & 20 & \\
\hline & & $\begin{array}{l}\text { Esmolol + } \\
\text { lidocaine (2) }\end{array}$ & $2 \mathrm{mg} / \mathrm{kg}+1.5 \mathrm{mg} / \mathrm{kg}$ & 20 & \\
\hline \multirow{2}{*}{$\begin{array}{l}\text { Hood D, } \\
1985^{23}\end{array}$} & United & Control & $\mathrm{N} / \mathrm{D}$ & 10 & \multirow{2}{*}{$\begin{array}{l}\text { MAP, HR, } \\
\text { Apgar }\end{array}$} \\
\hline & States & Nitroglycerin & $\begin{array}{l}200 \mathrm{mcg} / \mathrm{ml} \text { until BP } \\
\text { lowered } 20 \%\end{array}$ & 9 & \\
\hline $\begin{array}{l}\text { Kumar N, } \\
1993^{31}\end{array}$ & India & Control & $\begin{array}{l}\text { Capsule (similar } \\
\text { physical characteristic) }\end{array}$ & 15 & $\begin{array}{l}\text { HR, MAP, } \\
\text { Apgar }\end{array}$ \\
\hline
\end{tabular}

\begin{tabular}{|c|c|c|c|c|c|}
\hline $\begin{array}{l}\text { First author, } \\
\text { year }\end{array}$ & Country & Medication & Dosage & No. & Endpoints \\
\hline \multirow{3}{*}{$\begin{array}{l}\text { Park BY, } \\
2_{2011^{28}}\end{array}$} & \multirow{3}{*}{$\begin{array}{l}\text { South } \\
\text { Korea }\end{array}$} & Nifedipine & $\begin{array}{l}10 \mathrm{mg} \mathrm{PO}(20 \mathrm{~min} \text { before } \\
\text { induction) }\end{array}$ & 15 & \multirow{3}{*}{$\begin{array}{l}\text { SAP, HR, } \\
\text { Apgar }\end{array}$} \\
\hline & & $\begin{array}{l}\text { Remifentanil } \\
\text { (1) }\end{array}$ & $0.5 \mathrm{mcg} / \mathrm{kg}$ & 24 & \\
\hline & & $\begin{array}{l}\text { Remifentanil } \\
\text { (2) }\end{array}$ & $1 \mathrm{mcg} / \mathrm{kg}$ & 24 & \\
\hline \multirow{2}{*}{$\begin{array}{l}\text { Pournajafian } \\
\text { A, 2012 }\end{array}$} & \multirow{2}{*}{ Iran } & Remifentanil & $0.5 \mathrm{mcg} / \mathrm{kg} / \mathrm{min}$ & 20 & \multirow{2}{*}{$\begin{array}{l}\text { SAP, DAP, } \\
\text { HR }\end{array}$} \\
\hline & & Fentanyl & $50 \mathrm{mcg}$ & 18 & \\
\hline \multirow{2}{*}{$\begin{array}{l}\text { Ramanathan } \\
\text { J, 1988 }\end{array}$} & United & Control & $\mathrm{N} / \mathrm{D}$ & 10 & \multirow{2}{*}{$\begin{array}{l}\text { MAP, HR, } \\
\text { Apgar R }\end{array}$} \\
\hline & States & Labetalol & $\begin{array}{l}20 \mathrm{mg} \rightarrow 10 \mathrm{mg} \\
\text { increment every } 2 \mathrm{~min}\end{array}$ & 10 & \\
\hline \multirow{2}{*}{$\begin{array}{l}\text { Rout CC, } \\
1990^{27}\end{array}$} & South & Fentanyl & $2.5 \mathrm{mcg} / \mathrm{kg}$ & 20 & \multirow{2}{*}{$\begin{array}{l}\text { HR, SAP, } \\
\text { DAP, Apgar }\end{array}$} \\
\hline & Africa & Alfentanil & $10 \mathrm{mcg} / \mathrm{kg}$ & 20 & \\
\hline \multirow{3}{*}{$\begin{array}{l}\text { Safavi M, } \\
\text { 201133 }^{33}\end{array}$} & Iran & Hydralazine & $5-10 \mathrm{mg}$ IV & 40 & \multirow{3}{*}{$\begin{array}{l}\text { SAP, MAP, } \\
\text { DAP, HR, } \\
\text { Apgar }\end{array}$} \\
\hline & & Nitroglycerin & $\begin{array}{l}5 \mathrm{mcg} / \mathrm{min} \mathrm{IV} \\
\text { continuous }\end{array}$ & 40 & \\
\hline & & Nifedipine & $10 \mathrm{mg}$ sublingual & 40 & \\
\hline \multirow{2}{*}{$\begin{array}{l}\text { Yoo KY, } \\
20099^{34}\end{array}$} & South & Control & N/D & 21 & \multirow{4}{*}{$\begin{array}{l}\text { SAP, MAP, } \\
\text { DAP, HR, } \\
\text { Apgar } \\
\text { SAP, HR, } \\
\text { BIS, Apgar }\end{array}$} \\
\hline & Korea & Remifentanil & $1.0 \mathrm{mcg} / \mathrm{kg}$ & 21 & \\
\hline \multirow[t]{5}{*}{$\begin{array}{l}\text { Yoo KY, } \\
2013^{29}\end{array}$} & \multirow[t]{5}{*}{$\begin{array}{l}\text { South } \\
\text { Korea }\end{array}$} & $\begin{array}{l}\text { Remifentanil } \\
\text { (1) }\end{array}$ & $0.25 \mathrm{mcg} / \mathrm{kg}$ & 15 & \\
\hline & & $\begin{array}{l}\text { Remifentanil } \\
\text { (2) }\end{array}$ & $0.5 \mathrm{mcg} / \mathrm{kg}$ & 15 & \\
\hline & & $\begin{array}{l}\text { Remifentanil } \\
\text { (3) }\end{array}$ & $0.75 \mathrm{mcg} / \mathrm{kg}$ & 15 & \\
\hline & & $\begin{array}{l}\text { Remifentanil } \\
\text { (4) }\end{array}$ & $1.0 \mathrm{mcg} / \mathrm{kg}$ & 15 & \\
\hline & & $\begin{array}{l}\text { Remifentanil } \\
\text { (5) }\end{array}$ & $1.25 \mathrm{mcg} / \mathrm{kg}$ & 15 & \\
\hline
\end{tabular}

No., number of patients; Mg, magnesium; $\mathrm{SAP}$, systolic arterial pressure; MAP, mean arterial pressure; DAP, diastolic arterial pressure; HR, heart rate; Apgar, Apgar score; BIS, bispectral index; N/D = not defined.

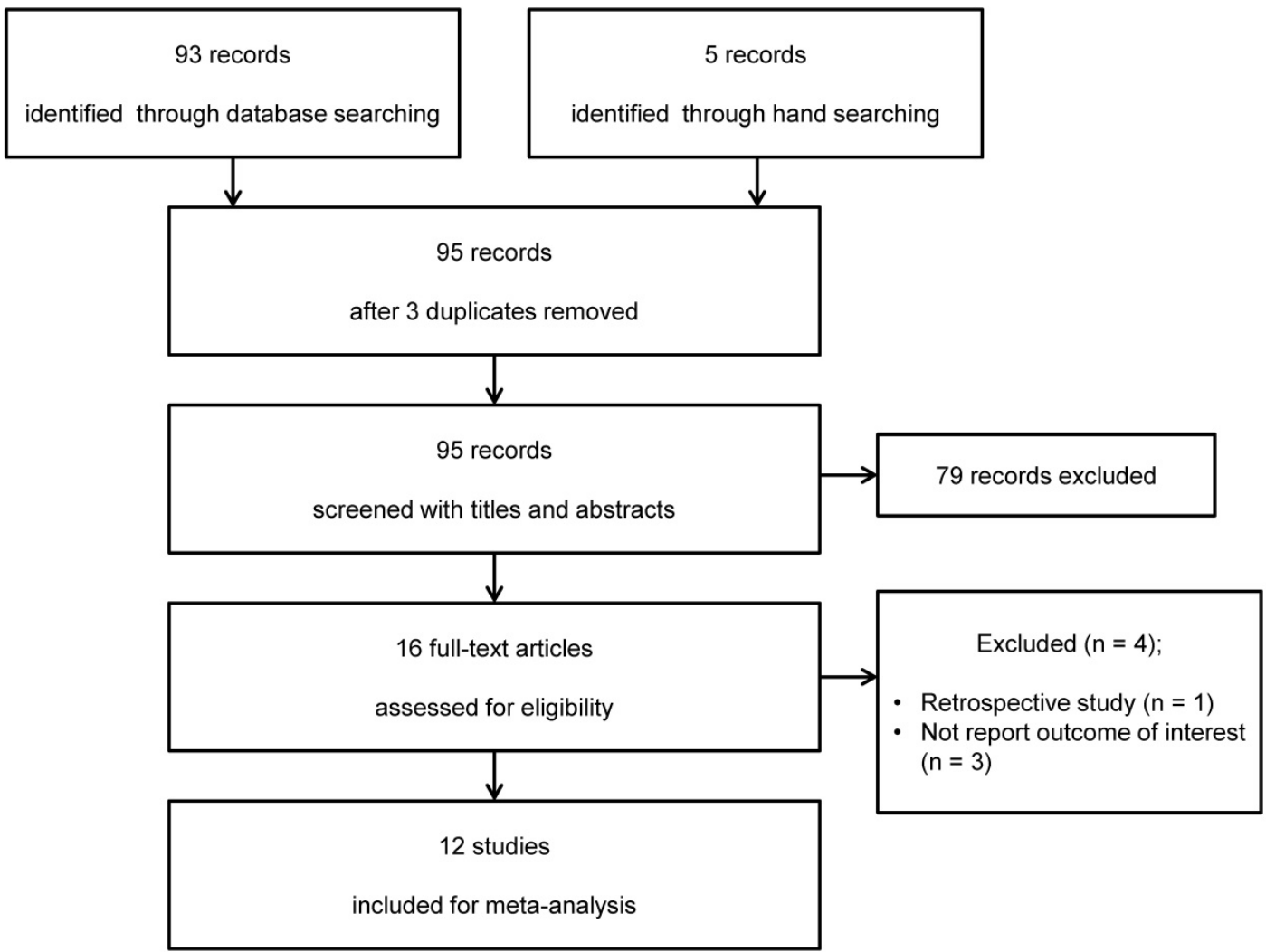

Figure 1. PRISMA flowchart of trial inclusion and exclusion. 


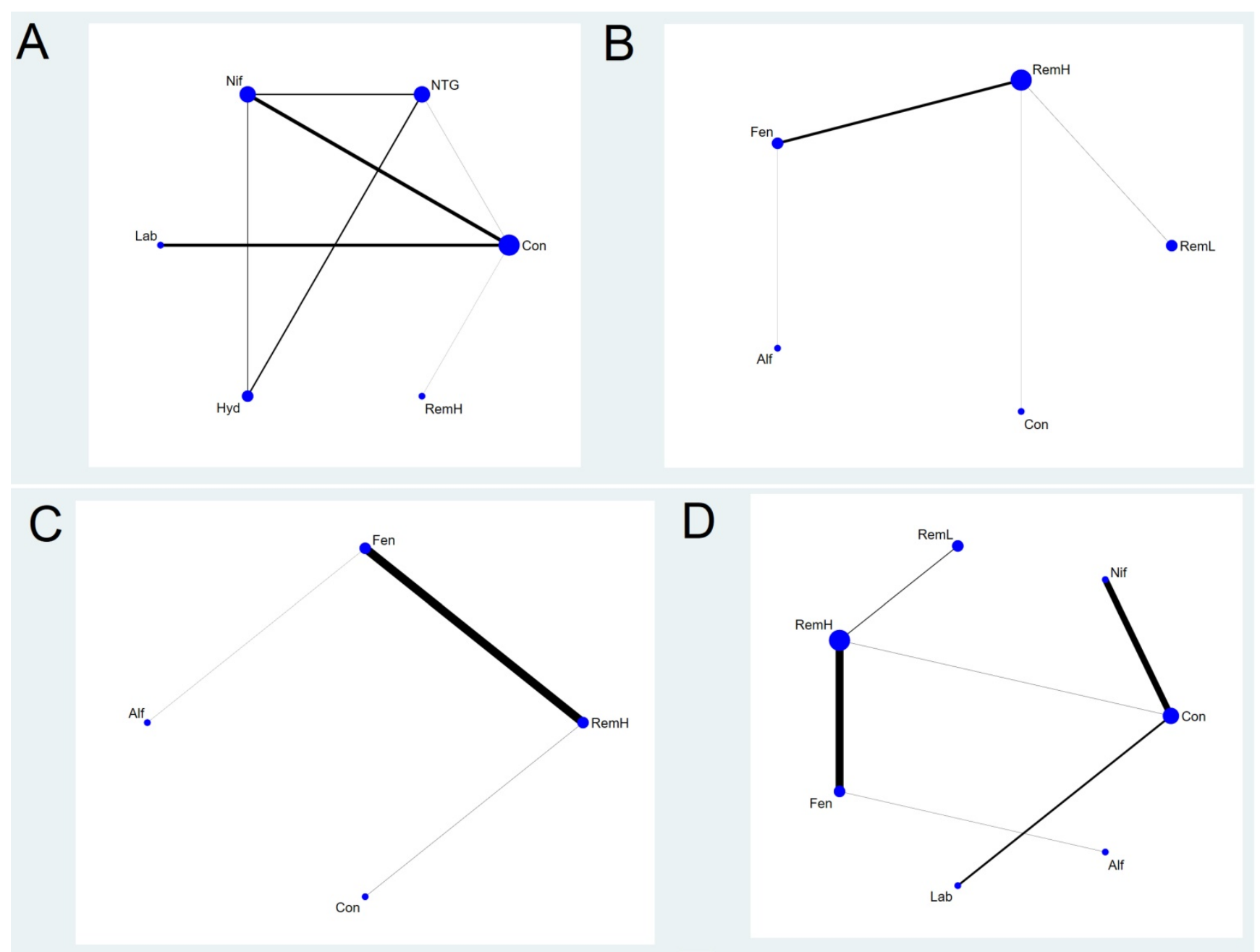

Figure 2. Network plot of the included studies comparing different pharmacological strategies. The nodes represent the pharmacological regimens used to prevent haemodynamic changes after intubation in parturient women with hypertensive disorders of pregnancy, and the edges show the available direct comparisons among them. Nodes and edges are weighted on the basis of the number of patients included and the inverse standard error of the effect. A) maximal mean arterial pressure, B) maximal systolic arterial pressure, C) maximal diastolic arterial pressure, D) maximal heart rate. Alf, alfentanil; Con, control; Fen, fentanyl; Hyd, hydralazine; Lab, labetalol; Nif, nifedipine; NTG, nitroglycerin; RemH, high dose of remifentanil; RemL, low dose of remifentanil.

\section{Maximal Mean Arterial Pressure}

Six pharmacological agents were compared in five studies (231 patients) for MMAP (Figure 2A) [23, $24,31,33,34]$. Seven comparisons were performed using mixed evidence (both direct and indirect evidence) and eight using indirect evidence alone (Supplementary Figure S1).

There were two closed loops in the network related to the comparison of the MMAP, but one loop (nitroglycerin-nifedipine-hydralazine) was formed only in a multi-arm trial [33]. There was no significant local inconsistency between direct and indirect point estimates (Supplementary Figure S2).

High doses of remifentanil (RemH) showed lower MMAP than those for hydralazine, labetalol, nifedipine, nitroglycerin and control; hydralazine showed lower MMAP than that for nifedipine and control; labetalol showed lower MMAP than that for nifedipine and control; nitroglycerin showed lower
MMAP than those for nifedipine and control; nifedipine showed lower MMAP than that for control in terms of their $95 \%$ CIs (Figure 3A). The rankogram and cumulative ranking plot showed that RemH had the lowest MMAP (Figures S3A and S4A). The expected mean rankings and SUCRA values for each pharmacological agent in Figure 4A were highest for RemH $(99.4 \%)$, followed by those for nitroglycerin $(73.6 \%)$, labetalol (60.9\%) and hydralazine (45.8\%).

\section{Maximal Systolic Arterial Pressure}

A total of eight studies (481 patients) considered MSAP. We excluded three studies from the NMA that were separated from the loops [26, 30,33]. Thus, a total of five studies (243 patients) were analysed $[27-29,32,34]$. The network plot of all eligible comparisons for this endpoint is depicted in Figure 2B. Four comparisons were performed using mixed evidence (both direct and indirect evidence) and six using indirect evidence alone (Supplementary Figure 
S1B). As there was no closed loop in the network, inconsistency was not evaluated.

RemH showed lower MSAP than those for RemL, fentanyl, and control; fentanyl showed lower MSAP than those for RemL and control; alfentanil showed lower MSAP than that for control, and RemL showed lower MSAP than that for control (Figure 3B).
The rankogram and cumulative ranking plot showed that RemH had the lowest MSAP (Figures S3B and S4B). The expected mean rankings and SUCRA values for each pharmacological agent (Figure 4B) were highest for RemH (92.6\%), followed by those for alfentanil $(67.9 \%)$ and fentanyl $(63.6 \%)$.

Table 2. Risk of bias

\begin{tabular}{|c|c|c|c|c|c|c|}
\hline First author, year & $\begin{array}{l}\text { Bias arising from the } \\
\text { randomisation process }\end{array}$ & $\begin{array}{l}\text { Bias due to deviations from } \\
\text { intended intervention }\end{array}$ & $\begin{array}{l}\text { Bias due to missing } \\
\text { outcome data }\end{array}$ & $\begin{array}{l}\text { Bias in measurement of } \\
\text { the outcome }\end{array}$ & $\begin{array}{l}\text { Bias in selection of the } \\
\text { reported results }\end{array}$ & $\begin{array}{l}\text { Overall } \\
\text { Bias }\end{array}$ \\
\hline Allen RW, $1991^{25}$ & Some concerns & Low risk & Low risk & Low risk & Low risk & $\begin{array}{l}\text { Some } \\
\text { concerns }\end{array}$ \\
\hline $\begin{array}{l}\text { Ashton WB, } \\
1991^{26}\end{array}$ & Some concerns & Low risk & Low risk & Low risk & Low risk & $\begin{array}{l}\text { Some } \\
\text { concerns }\end{array}$ \\
\hline Bansal S, $2005^{30}$ & Low risk & Low risk & Low risk & Low risk & Low risk & Low risk \\
\hline Hood D, $1985^{23}$ & Some concerns & Low risk & Low risk & Low risk & Low risk & $\begin{array}{l}\text { Some } \\
\text { concerns }\end{array}$ \\
\hline Kumar N $1993^{31}$ & Low risk & Low risk & Low risk & Low risk & Low risk & Low risk \\
\hline Park BY $2011^{28}$ & Low risk & Low risk & Low risk & Low risk & Low risk & Low risk \\
\hline $\begin{array}{l}\text { Pournajafian A } \\
2012^{32}\end{array}$ & Some concerns & Low risk & Low risk & Low risk & Low risk & $\begin{array}{l}\text { Some } \\
\text { concerns }\end{array}$ \\
\hline $\begin{array}{l}\text { Ramanathan J } \\
1988^{24}\end{array}$ & Some concerns & Low risk & Low risk & Low risk & Low risk & $\begin{array}{l}\text { Some } \\
\text { concerns }\end{array}$ \\
\hline Rout CC $1990^{27}$ & Some concerns & Low risk & Low risk & Low risk & Low risk & $\begin{array}{l}\text { Some } \\
\text { concerns }\end{array}$ \\
\hline Safavi M $2011^{33}$ & Low risk & Low risk & Low risk & Low risk & Low risk & Low risk \\
\hline Yoo KY 200934 & Low risk & Low risk & Low risk & Low risk & Low risk & Low risk \\
\hline Yoo KY $2013^{29}$ & Low risk & Low risk & Low risk & Low risk & Low risk & Low risk \\
\hline
\end{tabular}

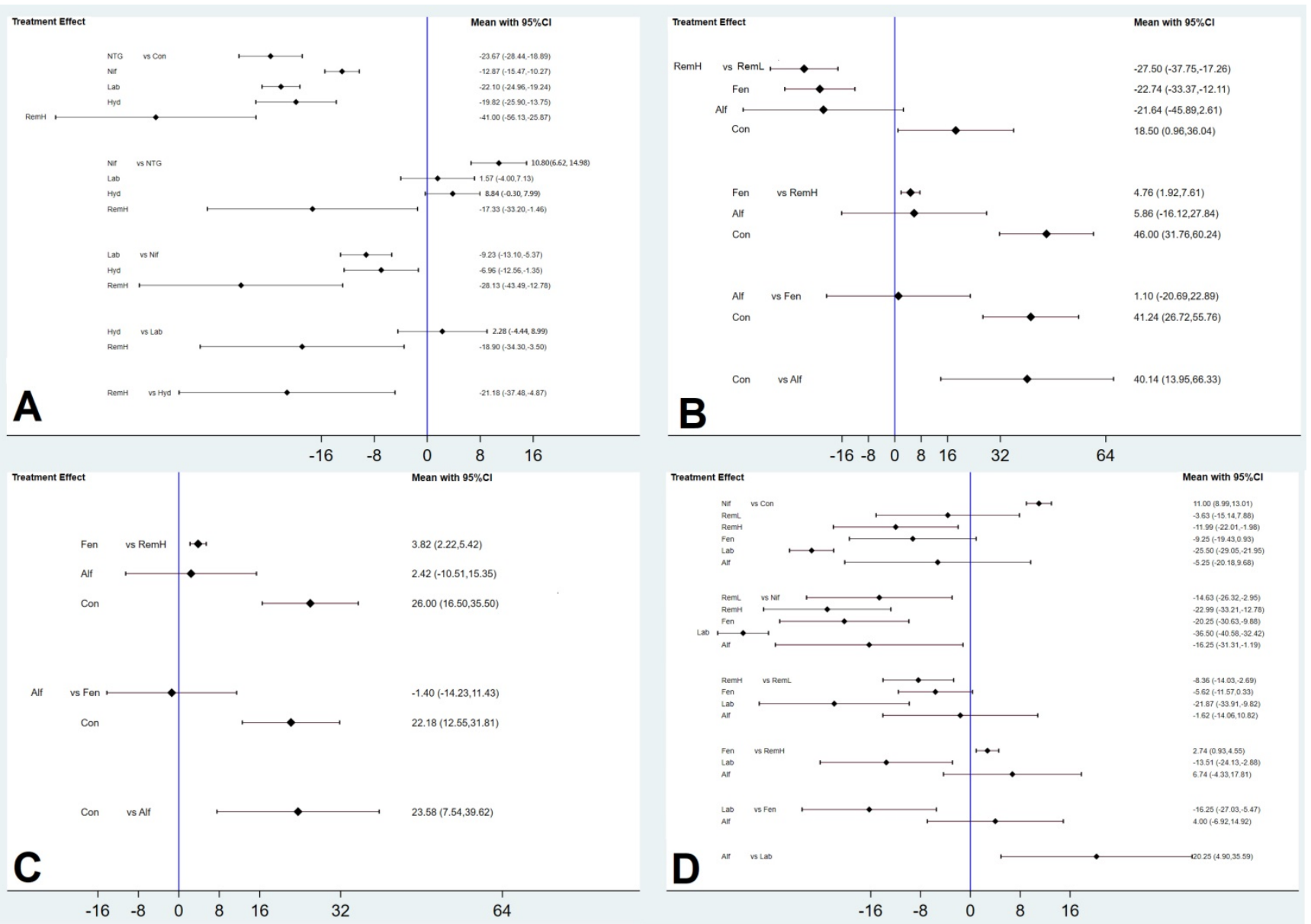

Figure 3. Confidence interval plots between each management modality and the placebo group. The diamond shape represents the mean summary effects; the black line, $95 \% \mathrm{Cl}$. A) maximal mean arterial pressure, B) maximal systolic arterial pressure, C) maximal diastolic arterial pressure, D) maximal heart rate. Cl, confidence interval; Alf, alfentanil; Con, control; Fen, fentanyl; Hyd, hydralazine; Lab, labetalol; Nif, nifedipine; NTG, nitroglycerin; RemH, high dose of remifentanil; RemL, low dose of remifentanil. 

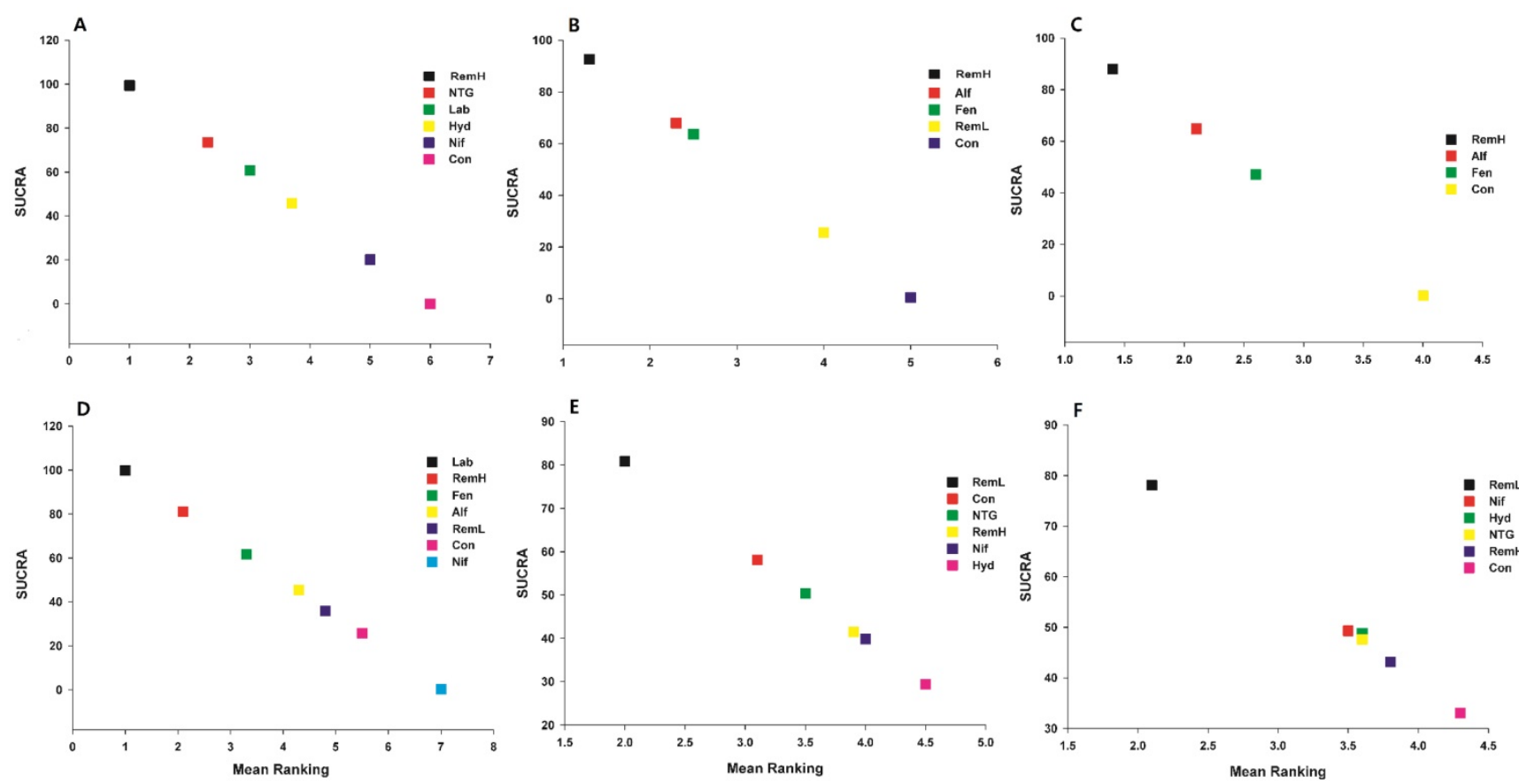

Figure 4. Expected mean ranking and surface under the cumulative ranking curve (SUCRA) values. The $X$-axis represents the expected mean ranking based on the SUCRA value, and the $Y$-axis represents the SUCRA value. A) maximal mean arterial pressure, B) maximal systolic arterial pressure, C) maximal diastolic arterial pressure, D) maximal heart rate, E) Apgar score at $1 \mathrm{~min}$, and F) Apgar score at 5 min. Alf, alfentanil; Con, control; Fen, fentanyl; Hyd, hydralazine; Lab, labetalol; Nif, nifedipine; NTG, nitroglycerin; RemH, high dose of remifentanil; RemL, low dose of remifentanil.

\section{Maximal Diastolic Arterial Pressure}

A total of four studies (240 patients) measured MDAP. We excluded one study from the NMA because it was separated from the loops [33]. Thus, a total of three studies (120 patients) were analysed [27, 32 , 34]. The network plot of all eligible comparisons for this endpoint is depicted in Figure 2C. Three comparisons were performed using mixed evidence (both direct and indirect evidence) and three using indirect evidence alone (Supplementary Figure S1C). As there was no closed loop in the network, inconsistency was not evaluated.

RemH showed lower MDAP than that for fentanyl and control; fentanyl showed lower MDAP than that for control; alfentanil showed lower MDAP than that for control (Figure 3C).

The rankogram and cumulative ranking plot showed that RemH had the lowest MDAP (Figures S3C and S4C). The expected mean rankings and SUCRA values for each pharmacological agent (Figure 4C) were highest for RemH (88.0\%), followed by those for alfentanil (64.8\%) and fentanyl (47.2\%).

\section{Maximal Heart Rate}

A total of 10 studies (531 patients) measured MHR. We excluded three studies from the NMA because they were separated from the loops [26, 30, 33]. Thus, a total of seven studies (293 patients) were analysed [24, 27-29, 31, 32, 34]. The network plot of all eligible comparisons for this endpoint is depicted in
Figure 2D. Six comparisons were performed using mixed evidence (both direct and indirect evidence) and 15 using indirect evidence alone (Supplementary Figure S1D). As there was no closed loop in the network, inconsistency was not evaluated.

Labetalol showed lower MHR than that for RemH, fentanyl, alfentanil, RemL, control, and nifedipine. RemH showed lower MHR than that for fentanyl, RemL, control, and nifedipine. Fentanyl showed lower MHR than that for nifedipine. Alfentanil showed lower MHR than that for nifedipine. Nifedipine showed higher MHR than that for control (Figure 3D).

The rankogram and cumulative ranking plot showed that labetalol had the lowest MHR (Figure S3D and S4D). The expected mean rankings and SUCRA values for each pharmacological agent (Figure 4D) were highest for labetalol (99.9\%), followed by those for RemH (81.2\%) and fentanyl $(61.6 \%)$.

\section{Apgar Score at 1 Minute}

A total of nine studies (468 patients) measured the Apgar score at $1 \mathrm{~min}$. Of those, seven reported the Apgar score at $1 \mathrm{~min}$ using a categorical variable (Apgar $\geq 7$ vs. Apgar < 7) [23, 27-30, 33, 34], while four studies reported it using a continuous variable $[26,28$, 29, 31]; two studies reported both [28, 29]. Thus, we analysed the Apgar score at $1 \mathrm{~min}$ using continuous or categorical variables separately. 
Considering the four studies (191 patients) that measured the Apgar score at $1 \mathrm{~min}$ reporting continuous variables, we performed pair-wise meta-analysis because two studies were separated from the loops [26, 31] and the remaining two compared two identical groups (RemL vs. RemH) [28, 29]. The Apgar score at $1 \mathrm{~min}$ was higher for RemL than for RemH (MD, 0.726; 95\% CI, 0.056 to 1.396; $\mathrm{I}^{2}=$ $0.0 \%)$.

Among the seven studies (281 patients) that reported the Apgar score at $1 \mathrm{~min}$ as a categorical variable, two were excluded from the NMA because they were separated from the loops $[27,30]$. Thus, a total of five studies (293 patients) were analysed [23, 28, 29, 33, 34]. The network plot of all eligible comparisons for this endpoint is depicted in Figure S5A.

One comparison (RemL vs. RemH) was conducted using direct evidence alone. Five comparisons were conducted using mixed evidence (both direct and indirect evidence) and nine using indirect evidence alone (Supplementary Figure S1E). As a closed loop (nitroglycerin-nifedipinehydralazine) was formed in the multi-arm trial [33], inconsistency was not evaluated. RemH showed lower Apgar score at $1 \mathrm{~min}$ than RemL (Figure S6A).

The rankogram and cumulative ranking plot showed that RemL had the lowest incidence of lower Apgar score (Apgar < 7) (Figures S3E and S4E). The expected mean rankings and SUCRA values of each pharmacological agent in Figure 4E were highest for RemL $(80.9 \%)$, followed by those for control (58.1\%) and nitroglycerin (50.4\%).

\section{Apgar Score at 5 minutes}

A total of nine studies (468 patients) measured the Apgar score at $5 \mathrm{~min}$. Of those, seven studies reported the Apgar score at $5 \mathrm{~min}$ as a categorical variable (Apgar $\geq 7$ vs. Apgar < 7) [23, 27-30, 33, 34], four reported it as a continuous variable[26, 28, 29, $31]$, and two reported both[28, 29]. Thus, we analysed the Apgar score at $5 \mathrm{~min}$ using continuous or categorical variables separately.

Considering the four studies (191 patients) that reported the Apgar score at $5 \mathrm{~min}$ as a continuous variable, we performed pair-wise meta-analysis because two studies were separated from the loops[26, 31] and the remaining two compared two identical groups (RemL vs. RemH)[28, 29]. There was no evidence of differences between the groups (MD, $0.359 ; 95 \% \mathrm{CI},-0.001$ to $0.720 ; \mathrm{I}^{2}=0.0 \%$ ).

Among the seven studies (281 patients) that reported the Apgar score at $5 \mathrm{~min}$ as a categorical variable, two that were separated from the loops were excluded from the NMA [27, 30]. Thus, a total of five studies (293 patients) were analysed [23, 28, 29, 33, 34]. The network plot of all eligible comparisons for this endpoint is depicted in Figure S5B.

One comparison (RemL vs. RemH) was conducted using direct evidence alone. Five comparisons were conducted using mixed evidence (both direct and indirect evidence) and nine using indirect evidence alone (Supplementary Figure S1F). As one closed loop (nitroglycerin-nifedipinehydralazine) was formed in the multi-arm trial [33], inconsistency was not evaluated. There was no evidence of differences between pharmacological agents (Figure S6B).

The rankogram and cumulative ranking plot showed that RemL had the lowest incidence of lower Apgar score (Apgar < 7; Figures 4F and S3F). The expected mean rankings and SUCRA values (Figure $4 \mathrm{~F})$ were highest for RemL (78.1\%), followed by those for nifedipine $(49.3 \%)$ and hydralazine $(48.8 \%)$.

Table 3. Grading of Recommendations, Assessment, Development, and Evaluation (GRADE) evidence quality for each outcome

\begin{tabular}{|c|c|c|c|c|c|c|c|}
\hline \multirow[t]{2}{*}{ Outcomes } & \multirow[t]{2}{*}{ No. of studies } & \multicolumn{5}{|c|}{ Quality assessment } & \multirow[t]{2}{*}{ Quality } \\
\hline & & Risk of bias & Inconsistency & Indirectness & Imprecision & Publication bias & \\
\hline Maximal MAP & 5 & not serious & not serious & not serious & serious & none & $\begin{array}{l}\bigoplus \oplus \bigoplus \bigcirc \\
\text { Moderate }\end{array}$ \\
\hline Maximal SAP & 8 & not serious & not serious & not serious & serious & none & $\begin{array}{l}\oplus \oplus \bigoplus \bigcirc \\
\text { Moderate }\end{array}$ \\
\hline Maximal DAP & 4 & not serious & not serious & not serious & serious & none & $\begin{array}{l}\oplus \bigoplus \bigoplus \bigcirc \\
\text { Moderate }\end{array}$ \\
\hline Maximal HR & 10 & not serious & not serious & not serious & serious & none & $\begin{array}{l}\bigoplus \oplus \bigoplus \bigcirc \\
\text { Moderate }\end{array}$ \\
\hline Apgar score at $1 \mathrm{~min}$ & 9 & not serious & not serious & not serious & serious & none & $\begin{array}{l}\oplus \oplus \bigoplus \bigcirc \\
\text { Moderate }\end{array}$ \\
\hline Apgar score at $5 \mathrm{~min}$ & 9 & not serious & not serious & not serious & serious & none & $\begin{array}{l}\bigoplus \oplus \bigoplus \bigcirc \\
\text { Moderate }\end{array}$ \\
\hline
\end{tabular}

$\mathrm{MAP}$, mean arterial pressure; SAP, systolic arterial pressure; DAP, diastolic arterial pressure; $\mathrm{HR}$, heart rate 


\section{Discussion}

Haemodynamic management in parturient women with hypertensive disorders of pregnancy undergoing caesarean section under general anaesthesia requires meticulous effort. These patients are more susceptible to hypertension and tachycardia after intubation, and physicians have adopted and compared several pharmacological strategies to avoid adverse outcomes such as intracranial pressure, cerebral haemorrhage, cardiac failure, and pulmonary oedema. To identify the most effective pharmacological strategy, we performed an NMA with 12 studies that compared arterial blood pressure, heart rate, and foetal outcomes.

Systolic, diastolic, and mean arterial blood pressures were compared between seven different pharmacological strategies-high and low doses of remifenatnil, alfentanil, fentanyl, nitroglycerin, hydralazine, labetalol, and nifedipine. Among these drugs, high dose of remifentanil produced the smallest changes in systolic, diastolic, and mean blood pressures and was therefore found to be the most effective drug in maintaining stable haemodynamic condition after intubation. Remifentanil's unique pharmacokinetic characteristics, due to its ultrashort effect and half-life, provided optimal analgesia and antihypertensive effects in women with hypertensive disorders of pregnancy. In addition, high dose of remifentanil $(1.0 \mathrm{mcg} / \mathrm{kg}, 1.25 \mathrm{mcg} / \mathrm{kg}$, and continuous infusion at a rate of $0.5 \mathrm{mcg} / \mathrm{kg} / \mathrm{min}, 3$ min prior to intubation) was significantly more effective than low dose of remifentanil $(0.25 \mathrm{mcg} / \mathrm{kg}$, $0.5 \mathrm{mcg} / \mathrm{kg}$, and $0.75 \mathrm{mcg} / \mathrm{kg}$ ) in controlling systolic arterial pressure. However, since delayed respiratory depression may occur in some newborns whose mothers are administered high dose of remifentanil, cautious dosing of this drug must be considered [35]. In addition, high dose of remifentanil may result in hypotension in parturient women, leading to decreased uterine blood flow. However, the differences in Apgar scores extracted from the NMA were not significant between the various doses of remifentanil, and the data were insufficient to analyse hypotension events among different pharmacological strategies.

In addition to arterial blood pressure, the heart rates of parturient women after intubation were compared among five pharmacological strategies high and low doses of remifentanil, labetalol, fentanyl, alfentanil, and nifedipine. In contrast with the results obtained for arterial blood pressure, labetalol was significantly most effective in maintaining baseline heart rate, followed by high doses of remifentanil and fentanyl. The $a$ and $\beta$ antagonistic effects of labetalol decrease both heart rate and blood pressure, and therefore, labetalol was more effective in controlling heart rate than other antihypertensive drugs. Tachycardia during induction may result in increased myocardial oxygen demand, and could be a risk factor for the development of myocardial ischaemia and infarction for patients with cardiovascular disease [36]. Hence, labetalol could be effective for the management of heart rate in high-risk hypertensive pregnant women.

The main concern regarding the use of opioids and antihypertensive drugs is their effects on newborns. When the Apgar scores as categorical variables at 1 and $5 \mathrm{~min}$ were compared between drugs, there was no evidence of differences, except that high dose of remifentanil resulted in significantly lower Apgar scores than low dose of remifentanil. Moreover, pair-wise meta-analysis comparing the Apgar scores as continuous variables at 1 and $5 \mathrm{~min}$ between low-dose and high-dose remifentanil showed no evidence of difference between the two groups. Although opioid use prior to delivery of the foetus is still controversial issue, we should be flexible on the change in favour of opioids administration which can be accompanied with greater benefits than not using them for both the mother and the foetus. In pregnant women with serious condition which is mandatory to maintain adequate haemodynamic state, the remifentanil can be a main option.

Although there was no evidence of significant differences in almost all comparisons and no cases of prolonged neurological adverse effects in the included studies, some studies reported respiratory depression, and sometimes tracheal intubation was required even in elective, uncomplicated term pregnancies $[35,37]$. As the birth weight of newborns decreases [38] and the incidence of respiratory distress syndrome increases in infants of mothers with hypertensive disorders of pregnancy [39], meticulous attention is required when applying pharmacological strategies to prevent haemodynamic changes after intubation in such mothers. Overall, the results of our NMA suggest that high dose of remifentanil reduces blood pressure during intubation in parturient women with hypertensive disorders of pregnancy.

There were several limitations in this study. First, as our NMA included 12 studies conducted in different clinical centres, methodological heterogeneity was present, and some study designs were not presented in sufficient detail. Additionally, some studies $[26,30,33]$ were separated from the loops and could not be compared; hence, the data concerning magnesium sulphate, esmolol, and lidocaine were excluded from this NMA. Well-designed, large scale RCTs that include various drugs should be conducted 
in the future to complement our study findings.

In conclusion, hypertension and tachycardia during intubation for general anaesthesia in patients with hypertensive disorders of pregnancy could be lethal. Our study showed that remifentanil and labetalol were the most effective treatments for the management of blood pressure and heart rate, respectively. However, their effects in newborns should be considered and the appropriate dosage of these drugs should be investigated in the future.

\section{Supplementary Material}

Supplementary figures.

http://www.medsci.org/v18p1039s1.pdf

\section{Acknowledgements}

\section{Author Contributions}

SWY and GJC equally contributed to this study; SWY and GJC designed the study, conducted the study (screened and selected the studies and extracted data), and wrote the manuscript; HKS conducted the study (helped select the studies and extract data), and provided critical revision of the manuscript; MJL conducted the study (helped select the studies, extract data, and assess the risk of bias), and provided critical revision of the manuscript; HK designed the study, conducted the study (extracted data), assessed the risk of bias, analysed and interpreted the data, and wrote the manuscript; all authors approved the final manuscript.

\section{Declaration of funding}

This research was supported by the Basic Science Research Program through the National Research Foundation of Korea (NRF) funded by the Ministry of Education, Science and Technology [grant no. 2020R1C1C1011263].

This research was supported by the Chung-Ang University Research Grants in 2019.

\section{Competing Interests}

The authors have declared that no competing interest exists.

\section{References}

1. King BD, Harris LC, Jr., Greifenstein FE, Elder JD, Jr., Dripps RD. Reflex circulatory responses to direct laryngoscopy and tracheal intubation performed during general anesthesia. Anesthesiology. 1951; 12: 556-66.

2. Low JM, Harvey JT, Prys-Roberts C, Dagnino J. Studies of anaesthesia in relation to hypertension. VII: Adrenergic responses to laryngoscopy. Br J Anaesth. 1986; 58: 471-7.

3. Prys-Roberts C, Meloche R, Foex P. Studies of anaesthesia in relation to hypertension. I. Cardiovascular responses of treated and untreated patients. Br J Anaesth. 1971; 43: 122-37.

4. Prys-Roberts C, Greene LT, Meloche R, Foex P. Studies of anaesthesia in relation to hypertension. II. Haemodynamic consequences of induction and endotracheal intubation. Br J Anaesth. 1971; 43: 531-47.

5. Lawes EG, Downing JW, Duncan PW, Bland B, Lavies N, Gane GA. Fentanyl-droperidol supplementation of rapid sequence induction in the presence of severe pregnancy-induced and pregnancy-aggravated hypertension. Br J Anaesth. 1987; 59: 1381-91.

6. Shamseer L, Moher D, Clarke M, Ghersi D, Liberati A, Petticrew M, et al. Preferred reporting items for systematic review and meta-analysis protocols (PRISMA-P) 2015: elaboration and explanation. BMJ (Clinical research ed). 2015; 350: g7647.

7. Yoon SW, Kang H, Choi GJ. Pharmacologic strategies to prevent hemodynamic changes after intubation in parturient women with hypertensive disorders of pregnancy: A systematic review and network meta-analysis protocol. Medicine. 2019; 98: e18454.

8. Cochrane handbook for systematic reviews of interventions.: Cochrane; 2019.

9. Cornell JE. The PRISMA extension for network meta-analysis: bringing clarity and guidance to the reporting of systematic reviews incorporating network meta-analyses. Annals of internal medicine. 2015; 162: 797-8.

10. Viera AJ, Garrett JM. Understanding interobserver agreement: the kappa statistic. Family medicine. 2005; 37: 360-3.

11. Higgins JP, Sterne JA, Savovic J, Page MJ, Hróbjartsson A, Boutron I, et al. A revised tool for assessing risk of bias in randomized trials. 2016; 10: 29-31.

12. Salanti G. Indirect and mixed-treatment comparison, network, or multiple-treatments meta-analysis: many names, many benefits, many concerns for the next generation evidence synthesis tool. Res Synth Methods. 2012; 3: 80-97.

13. Chaimani A, Higgins JP, Mavridis D, Spyridonos P, Salanti G. Graphical tools for network meta-analysis in STATA. PLoS One. 2013; 8.

14. White IR, Barrett JK, Jackson D, Higgins JP. Consistency and inconsistency in network meta-analysis: model estimation using multivariate meta-regression. Res Synth Methods. 2012; 3: 111-25.

15. Salanti G, Ades AE, Ioannidis JP. Graphical methods and numerical summaries for presenting results from multiple-treatment meta-analysis: an overview and tutorial. J Clin Epidemiol. 2011; 64: 163-71.

16. Riley RD, Higgins JP, Deeks JJ. Interpretation of random effects meta-analyses. BMJ (Clinical research ed). 2011; 342: d549.

17. Higgins JP, Thompson SG, Deeks JJ, Altman DG. Measuring inconsistency in meta-analyses. BMJ (Clinical research ed). 2003; 327: 557-60.

18. Atkins D, Best D, Briss PA, Eccles M, Falck-Ytter Y, Flottorp S, et al. Grading quality of evidence and strength of recommendations. BMJ (Clinical research ed). 2004; 328: 1490 .

19. Ananth CV, Lavery JA, Friedman AM, Wapner RJ, Wright JD. Serious maternal complications in relation to severe pre-eclampsia: a retrospective cohort study of the impact of hospital volume. BJOG : an international journal of obstetrics and gynaecology. 2017; 124: 1246-53.

20. Das M, Chaudhuri PR, Mondal BC, Mitra S, Bandyopadhyay D, Pramanik S. Assessment of serum magnesium levels and its outcome in neonates of eclamptic mothers treated with low-dose magnesium sulfate regimen. Indian journal of pharmacology. 2015; 47: 502-8

21. Salman MM, Goetze N, Badrinath M, Shah M. The videolaryngoscope as a first-line intubation device in women with hypertensive disease of pregnancy. International journal of obstetric anesthesia. 2018; 33: 87-8.

22. Eskandr AM, Metwally AA, Ahmed AA, Elfeky EM, Eldesoky IM, Obada MA, et al. Dexmedetomidine as a part of general anaesthesia for caesarean delivery in patients with pre-eclampsia: A randomised double-blinded trial. European journal of anaesthesiology. 2018; 35: 372-8.

23. Hood DD, Dewan DM, James FM, 3rd, Floyd HM, Bogard TD. The use of nitroglycerin in preventing the hypertensive response to tracheal intubation in severe preeclampsia. Anesthesiology. 1985; 63: 329-32.

24. Ramanathan J, Sibai BM, Mabie WC, Chauhan D, Ruiz AG. The use of labetalol for attenuation of the hypertensive response to endotracheal intubation in preeclampsia. American journal of obstetrics and gynecology. 1988; 159: 650-4.

25. Allen RW, James MF, Uys PC. Attenuation of the pressor response to tracheal intubation in hypertensive proteinuric pregnant patients by lignocaine, alfentanil and magnesium sulphate. Br J Anaesth. 1991; 66: 216-23.

26. Ashton WB, James MF, Janicki P, Uys PC. Attenuation of the pressor response to tracheal intubation by magnesium sulphate with and without alfentanil in hypertensive proteinuric patients undergoing caesarean section. Br J Anaesth. 1991; 67: 741-7.

27. Rout CC, Rocke DA. Effects of alfentanil and fentanyl on induction of anaesthesia in patients with severe pregnancy-induced hypertension. Br J Anaesth. 1990; 65: 468-74

28. Park BY, Jeong CW, Jang EA, Kim SJ, Jeong ST, Shin MH, et al. Dose-related attenuation of cardiovascular responses to tracheal intubation by intravenous remifentanil bolus in severe pre-eclamptic patients undergoing Caesarean delivery. Br J Anaesth. 2011; 106: 82-7.

29. Yoo KY, Kang DH, Jeong H, Jeong CW, Choi YY, Lee J. A dose-response study of remifentanil for attenuation of the hypertensive response to laryngoscopy and tracheal intubation in severely preeclamptic women undergoing caesarean delivery under general anaesthesia. International journal of obstetric anesthesia. 2013; 22: 10-8.

30. Bansal S, Pawar M. Haemodynamic responses to laryngoscopy and intubation in patients with pregnancy-induced hypertension: effect of intravenous esmolol with or without lidocaine. International journal of obstetric anesthesia. 2002; 11: 4-8.

31. Kumar N, Batra YK, Bala I, Gopalan S. Nifedipine attenuates the hypertensive response to tracheal intubation in pregnancy-induced hypertension. Canadian journal of anaesthesia $=$ Journal canadien d'anesthesie. 1993; 40: 329-33. 
32. Pournajafian A, Rokhtabnak F, Kholdbarin A, Ghodrati M, Ghavam S. Comparison of remifentanil and fentanyl regarding hemodynamic changes due to endotracheal intubation in preeclamptic parturient candidate for cesarean delivery. Anesthesiology and pain medicine. 2012; 2: 90-3.

33. Safavi M, Honarmand A, Azari N. Attenuation of the pressor response to tracheal intubation in severe preeclampsia: relative efficacies of nitroglycerine infusion, sublingual nifedipine, and intravenous hydralazine. Anesthesiology and pain medicine. 2011; 1: 81-9.

34. Yoo KY, Jeong CW, Park BY, Kim SJ, Jeong ST, Shin MH, et al. Effects of remifentanil on cardiovascular and bispectral index responses to endotracheal intubation in severe pre-eclamptic patients undergoing Caesarean delivery under general anaesthesia. Br J Anaesth. 2009; 102: 812-9.

35. Ngan Kee WD, Khaw KS, Ma KC, Wong AS, Lee BB, Ng FF. Maternal and neonatal effects of remifentanil at induction of general anesthesia for cesarean delivery: a randomized, double-blind, controlled trial. Anesthesiology. 2006; 104: 14-20.

36. Kanchi M, Nair HC, Banakal S, Murthy K, Murugesan C. Haemodynamic response to endotracheal intubation in coronary artery disease: Direct versus video laryngoscopy. Indian J Anaesth. 2011; 55: 260-5

37. Draisci G, Valente A, Suppa E, Frassanito L, Pinto R, Meo F, et al. Remifentanil for cesarean section under general anesthesia: effects on maternal stress hormone secretion and neonatal well-being: a randomized trial. International journal of obstetric anesthesia. 2008; 17: 130-6.

38. Lei F, Liu D, Shen Y, Zhang L, Li S, Liu X, et al. Study on the influence of pregnancy-induced hypertension on neonatal birth weight. Journal of investigative medicine : the official publication of the American Federation for Clinical Research. 2018; 66: 1008-14.

39. Bowen JR, Leslie GI, Arnold JD, Jones MP, Gallery ED. Increased incidence of respiratory distress syndrome in infants following pregnancies complicated by hypertension. The Australian \& New Zealand journal of obstetrics \& gynaecology. 1988; 28: 109-12. 